\title{
Potential of Biostimulants Based on PGPR Rhizobacteria Native to Benin's Soils on the Growth and Yield of Maize (Zea mays L.) under Greenhouse Conditions
}

\author{
Marcel Y. Adoko', Haziz Sina', Olaréwadjou Amogou', Nadège A. Agbodjato', \\ Pacôme A. Noumavo1,2, Ricardos M. Aguégué1, Sylvestre A. Assogba ${ }^{1}$, Nestor Ahoyo Adjovi ${ }^{3}$, \\ Gustave Dagbénonbakin ${ }^{3}$, Adolphe Adjanohoun3 ${ }^{3}$, Lamine Baba-Moussa1*
}

${ }^{1}$ Laboratory of Biology and Molecular Typing in Microbiology, Department of Biochemistry and Cellular Biology, University of Abomey Calavi (UAC), Cotonou, Benin

${ }^{2}$ Laboratory of Microbiology and Food Technology, Department of Plant Biology, University of Abomey Calavi (UAC), Cotonou, Benin

${ }^{3}$ National Agricultural Research Institute of Benin (INRAB), Benin

Email: *laminesaid@yahoo.fr

How to cite this paper: Adoko, M.Y., Sina, H., Amogou, O., Agbodjato, N.A., Noumavo, P.A., Aguégué, R.M., Assogba, S.A., Adjovi, N.A., Dagbénonbakin, G., Adjanohoun, A. and Baba-Moussa, L. (2021) Potential of Biostimulants Based on PGPR Rhizobacteria Native to Benin's Soils on the Growth and Yield of Maize (Zea mays L.) under Greenhouse Conditions. Open Journal of Soil Science, 11, 177-196.

https://doi.org/10.4236/ojss.2021.113010

Received: February 26, 2021

Accepted: March 22, 2021

Published: March 25, 2021

Copyright $\odot 2021$ by author(s) and Scientific Research Publishing Inc. This work is licensed under the Creative Commons Attribution International License (CC BY 4.0).

http://creativecommons.org/licenses/by/4.0/

\section{(c) (i) Open Access}

\begin{abstract}
The application of biostimulants in agriculture represents an environmentally friendly alternative while increasing agricultural production. The aims of the study were to develop solid biostimulants based on five rhizobacteria native to Benin's soils and to evaluate their efficacy on the growth and biomass yield of maize under greenhouse conditions on ferrallitic and ferruginous soils. Clay and peat were used as a conservation binder for the preparation of the biostimulants. These binders were used alone or combined in the different formulations with maize flour and sucrose. $10 \mathrm{~g}$ of biostimulants were applied at sowing in pots containing five kilograms of sterilised soil. The experimental design was a completely randomised block of 24 treatments with three replicates. The results obtained showed significant improvements $(P<0.001)$ in height (49.49\%), stem diameter (32.7\%), leaf area (66.10\%), above-ground biomass (97.12\%) and below-ground biomass (53.98\%) on ferrallitic soil with the application of the clay + Pseudomonas putida biostimulant compared to the control. On the other hand, the use of the peat biostimulant + Pseudomonas syringae was more beneficial for plant growth on ferruginous soil. The height, stem diameter, leaf area, above-ground biomass and below-ground biomass of the plants under the influence of this biostimulant were improved by $83.06 \%, 44.57 \%, 102.94 \%, 86.84 \%$ and $42.68 \%$, respectively, compared to the control. Therefore, these results confirm that Rhizobacteria express their
\end{abstract}


potential through biostimulants formulated on maize. The formulated biostimulants can later be used by producers to improve crop productivity for sustainable agriculture.

\section{Keywords}

Biostimulants, Plant Growth Promoting Rhizobacteria, Soil Fertility, Binder, Corn, Benin

\section{Introduction}

Maize is a staple food crop for many people in West African countries. In Benin, it is the leading cereal crop, accounting for $52.6 \%$ of total land area and $78.3 \%$ of all cereal production [1]. It is also used for animal feed and industrial uses in the form of starch, flour, ethanol and cooking syrup [2]. More than 90 maize-based foods and drinks have been identified and documented by Adjadi et al., 2015 [3]. Despite the importance of this speculation for food security, its productivity faces many constraints, including the constant decline in the fertility of cultivated land due to its degradation [4]. This situation forces producers to make heavy use of chemical inputs. The high use of these products causes them to accumulate in soils and food products, thus leading to an imbalance in the nutrient cycle [5]. This is an ecological concern whose consequences are felt by the population. It is imperative to find a realistic, cheap, better and safer way to maximise agricultural productivity. The application of biostimulants in crop production is an important ecological alternative to achieve all these criteria [6]. A plant biostimulant is a substance or micro-organism applied to plants to improve the nutritional efficiency, tolerance to abiotic stress and/or quality characteristics of crops, regardless of their nutrient content. The use of microorganisms as biostimulants is not a widespread practice. This is due to the particular conditions of use of each microbial group [7]. The application of biostimulants makes it possible to reduce the use of chemicals that have generated a serious environmental impact [8] [9].

In Benin, several studies have been carried out using inocula of PGPR rhizobacteria native to Benin soils to improve maize growth and yield [10] [11]. In the light of these studies, several strains have shown their effectiveness and in particular P. putida in controlled environments [12] [13] [14] and in farming environments [10]. During this work, the inoculation of bacterial suspension was a real problem raised by these different authors. Moreover, the conservation of the liquid biostimulant in natural conditions was also a difficulty for the authors as well as the growers who hosted the trials. Further research is needed to formulate effective biostimulants that can help us overcome these constraints. The aims of the study were to develop solid biostimulants based on five rhizobacteria native to Benin's soils and to evaluate their efficacy on the growth and biomass yield of maize under greenhouse conditions on ferrallitic and ferruginous soils. 


\section{Materials and Methods}

\subsection{Biological Material}

The rhizobacteria that were used are Pseudomonas putida (South), isolated and characterized from the rhizosphere of maize from the different development poles of Southern Benin by [15]; Pseudomonas putida (North), Pseudomonas syringae, Bacillus thuringiensis and Serratia marcescens isolated and characterized from the corn rhizosphere of different development poles of Central and Northern Benin by [16]. These rhizobacteria were kept at the Laboratory of Biology and Molecular Typing in Microbiology in Muller Hinton broth with added glycerol $(10 \%)$ at $-85^{\circ} \mathrm{C}$. The 2000 SYNEE maize variety supplied by National Agricultural Research Institute of Benin (INRAB) was used. It is an extra-early variety of 75 days, with a potential grain yield varying between 2 and $2.5 \mathrm{t} / \mathrm{ha}$ in rural areas [17]. Substrates such as peat and clay were used as binders in the preparation of the formulation.

\subsection{Validation of the Best Binder}

Starch, peat and clay were used to formulate the various biostimulants. These binders were sterilised using an autoclave at a temperature of $121^{\circ} \mathrm{C}$ for $30 \mathrm{~min}$ utes. After the formulation, the evolution of the microorganisms was followed in boxes incubated at $25^{\circ} \mathrm{C}$ for two months in an aseptic medium after which the strains were observed. The best binder was the one that maintained the bacteria during this time.

\subsection{Development of Biostimulants}

The adapted method of Connick et al., 1991 [18] has been used for the preparation of microbial biostimulants. For this purpose, $32 \mathrm{~g}$ maize flour, $30 \mathrm{ml}$ bacterial suspension $\left(10^{8} \mathrm{CFU} / \mathrm{ml}\right), 6 \mathrm{~g}$ binder and $2 \mathrm{~g}$ sucrose were filled into plastic boxes and mixed well with gloved hands under aseptic conditions until a soft dough was obtained. The control formulation was prepared with $32 \mathrm{~g}$ maize flour, $30 \mathrm{ml}$ sterile distilled water, $6 \mathrm{~g}$ binder and $2 \mathrm{~g}$ sucrose. After mixing, the different formulations were spread on aluminium foil for two days at a temperature of $25^{\circ} \mathrm{C}$ in an aseptic environment. After three days of drying in ambient air in an aseptic environment, the paste was crushed in a mortar and sieved.

\subsection{Chemical Analysis of Soils}

The soils used were sampled at $0-20 \mathrm{~cm}$ horizon using a marked shovel spade at the experimental sites. The ferrallitic soil was collected at the Agricultural Research Centre-South Niaouli and the ferruginous soil was collected in central Benin at the Reasearch \& Development site of Miniffi in Dassa-Zoumè district. The chemical analyses were carried out at the Laboratory of Soil, Water and Environmental Sciences of INRAB in order to determine their characteristics. The chemical analyses consisted of the determination of carbon and organic matter by the [19]; water $\mathrm{pH}$ and $\mathrm{KCl} \mathrm{pH}$ using a $\mathrm{pH}$ meter with $(1 / 2.5)$ as soil-water 
ratio; assimilable phosphorus by the [20]; exchangeable cations $(\mathrm{Ca}, \mathrm{Mg}, \mathrm{K}$ and $\mathrm{Na}$ ) by the ammonium acetate method using atomic absorption spectrophotometry [21].

\subsection{Experimental Device}

The experimental device was a randomized block of 24 treatments with three replicates per soil type. After sieving these soils, they were sterilized in an oven at a temperature of $120^{\circ} \mathrm{C}$ for 30 minutes and then introduced into plastic jars of 5 $\mathrm{dm}^{3}$ volume. The different treatments were defined as follows: $\mathrm{T}_{0}$ : control (without $\mathrm{PGPR}$ ), $\mathrm{T}_{1}:$ P. putida $S, \mathrm{~T}_{2}: P$. putida $\mathrm{N}, \mathrm{T}_{3}: P$. syringae, $\mathrm{T}_{4}: B$. thuringiensis, $\mathrm{T}_{5}: S$. marcescens, $\mathrm{T}_{6}$ : clay-peat (without PGPR), $\mathrm{T}_{7}$ : clay-peat $+P$. putida $\mathrm{S}, \mathrm{T}_{8}$ : clay-peat $+P$. putida $\mathrm{N}, \mathrm{T}_{9}$ : clay-peat $+P$. syringae, $\mathrm{T}_{10}$ : clay-peat + B. thuringiensis, $\mathrm{T}_{11}$ : clay-peat $+S$. marcescens, $\mathrm{T}_{12}$ : peat (without PGPR), $\mathrm{T}_{13}$ : peat $+P$. putida $\mathrm{S}, \mathrm{T}_{14}:$ peat $+P$. putida $\mathrm{N}, \mathrm{T}_{15}$ : peat $+P$. syringae, $\mathrm{T}_{16}$ : peat $+B$. thuringiensis, $\mathrm{T}_{17}$ : peat $+S$. marcescens, $\mathrm{T}_{18}$ : clay (without PGPR), $\mathrm{T}_{19}$ : clay $+P$. putida $\mathrm{S}, \mathrm{T}_{20}$ : clay $+P$. putida $\mathrm{N}, \mathrm{T}_{21}$ : clay $+P$. syringae, $\mathrm{T}_{22}$ : clay $+B$. thuringiensis, $\mathrm{T}_{23}$ : clay $+S$. marcescens.

\subsection{Sowing and Application of Biostimulant}

The different strains were applied individually to each type of soil in the form of a bacterial suspension at a rate of $10 \mathrm{ml}\left(10^{8} \mathrm{CFU} / \mathrm{ml}\right)$. In the control pots, $10 \mathrm{ml}$ of sterile distilled water was used instead of the bacterial suspension. Following the opening of the approximately $5 \mathrm{~cm}$ deep holes, two maize seeds were inserted and the holes were immediately closed again; then $10 \mathrm{~g}$ of formulated $\mathrm{mi}$ crobial biostimulant was applied according to the treatments and mixed with the upper part of the soil in the pots. The control pots received $10 \mathrm{~g}$ of control formulations.

\subsection{Data Collection}

The height and stem diameter of the plants were measured every 72 hours, on the 10th, 13th, 16th, 19th, 22nd, 25th, 28th and 31st days after sowing (DAS). The height (distance between the crown and the last ligule) of the maize plant was measured with a tape measure; the stem diameter was measured with a sliding foot. The leaf area was measured only on day 31 after sowing. It was estimated by the product of leaf length and width affected by the 0.75 coefficient [22]. The aerial and underground biomass were collected by treatment and by repetition and stored in an envelope designed for this purpose. The envelopes were placed in a $65^{\circ} \mathrm{C}$ oven for 72 hours until constant dry weight was obtained [23]. The weights were taken using a precision balance.

\subsection{Statistical Analysis}

The analyses were performed with R 3.6.0 software [24] using nlme packages [25], lsmeans, car, lattice, ggplot2, FctoMine R and factoextra [26]. Normality 
and homoscedasticity of the data were verified using Ryan-Joiner and Levene tests respectively [27] prior to performing ANOVA. Post-hoc or multiple comparison (SNK) tests were performed to assess statistical differences. The hierarchical classification of main components (HCPC) [28] was performed in order to identify the model of discrimination of treatments taking into account their performance. A Major Component Analysis (PCA) [29] [30] and a hierarchical classification were performed on these variables to classify treatments into homogeneous groups. The combination of these two analyses is useful to show the pattern across the data [31].

\section{Results}

\subsection{Validation of the Best Binder}

Two months after formulation, the biostimulants formulated with Serratia marcescens were presented in the different aspects of Figure 1. These strains had grown well in clay formulations, moderately with peat and very weakly with cooked starch. This growth was observed by the red coloration on these biostimulants. Clay and peat were the best binders.

\subsection{Chemical Characteristics of Soils}

The chemical properties of the two soil types before the tests were installed (Table 1) generally showed that the soil in Niaouli (ferrallitic) was slightly acidic and that in Miniffi (ferruginous) was alkaline. These soils showed a low level of fertility characterised by high $\mathrm{C} / \mathrm{N}$ ratios. The Miniffi soil was richer in exchangeable $\mathrm{K}+$ than the Niaouli soil. However, this soil had a low level of exchangeable organic carbon, assimilable phosphorus, $\mathrm{Ca}^{2+}$ and $\mathrm{Mg}^{2+}$.

\subsection{Evolution of the Height of Maize Plants on Ferrallitic Soil}

The evolution over time of the height of maize plants on ferrallitic soil is illustrated by the curves in Figure 2. The best height was recorded with the solid 31 DAS.

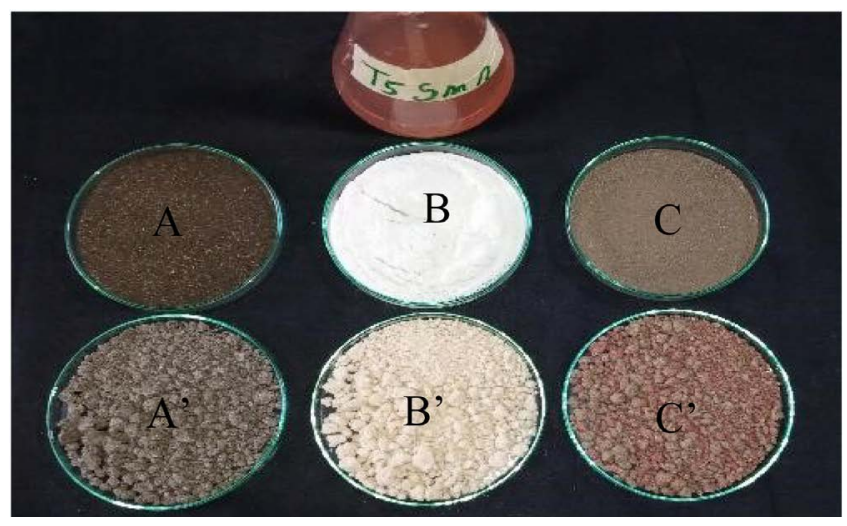

Figure 1. Appearance of Serratia marcescens biostimulants after two months. A: peat; A': peat $+S$. marcescens; B: starch; B': starch + S. marcescens; C: clay; C': clay + S. marcescens. 


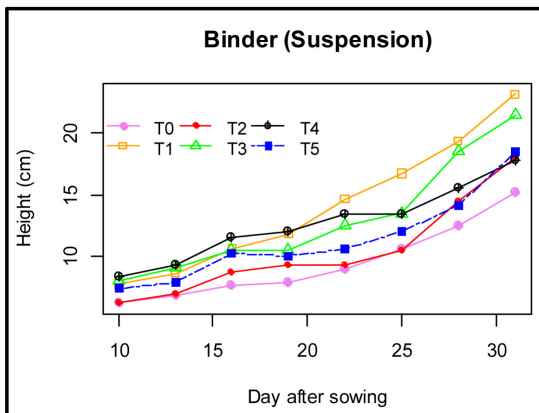

(a)

Binder (Peat)

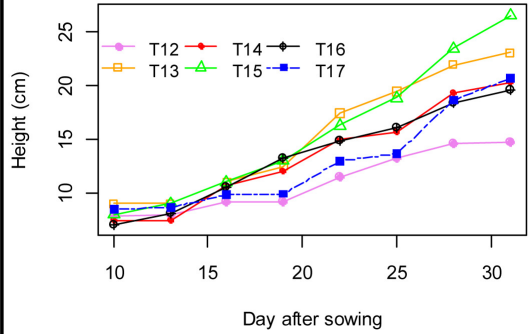

(c)

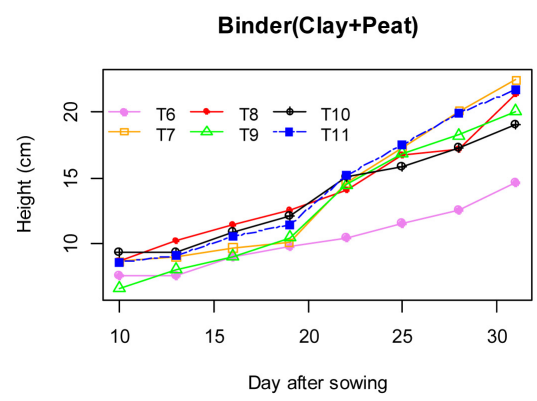

(b)

Binder (Clay)

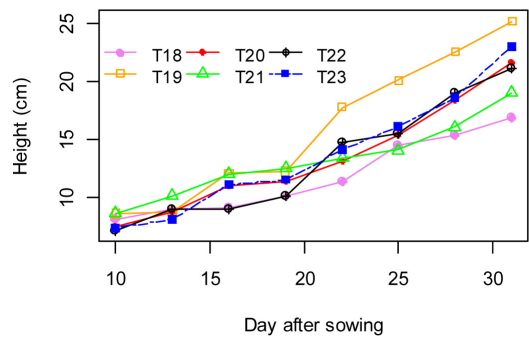

(d)

Figure 2. Effect of biostimulants on the height of maize plants on ferrallitic soil. (a) Suspension, (b) Biostimulant based on clay-peat, (c) Biostimulant based on peat, (d) Biostimulant based on clay. $\mathrm{T}_{0}$ : control, $\mathrm{T}_{1}:$ P. putida $\mathrm{S}, \mathrm{T}_{2}: P$. putida $\mathrm{N}, \mathrm{T}_{3}: P$. syringae, $\mathrm{T}_{4}: B$. thuringiensis, $\mathrm{T}_{5}: S$. marcescens, $\mathrm{T}_{6}$ : clay-peat (without PGPR), $\mathrm{T}_{7}$ : clay-peat + P. putida $\mathrm{S}$, $\mathrm{T}_{8}$ : clay-peat $+P$. putida $\mathrm{N}, \mathrm{T}_{9}$ : clay-peat $+P$. syringae, $\mathrm{T}_{10}$ : clay-peat $+B$. thuringiensis, $\mathrm{T}_{11}$ : peat-clay $+S$. marcescens, $\mathrm{T}_{12}:$ peat (without $\mathrm{PGPR}$ ), $\mathrm{T}_{13}:$ peat $+P$. putida $\mathrm{S}, \mathrm{T}_{14}:$ peat + P. putida $\mathrm{N}, \mathrm{T}_{15}$ : peat + P. syringae, $\mathrm{T}_{16}$ : peat + B. thuringiensis, $\mathrm{T}_{17}:$ peat $+S$. marcescens, $\mathrm{T}_{18}$ : clay (without PGPR), $\mathrm{T}_{19}$ : clay $+P$. putida $\mathrm{S}, \mathrm{T}_{20}$ : clay $+P$. putida $\mathrm{N}, \mathrm{T}_{21}$ : clay + $P$. syringae, $\mathrm{T}_{22}$ : clay $+B$. thuringiensis, $\mathrm{T}_{23}$ : clay $+S$. marcescens.

Table 1. Chemical properties of soils prior to test installation.

\begin{tabular}{|c|c|c|c|c|c|c|c|c|c|c|}
\hline \multirow{2}{*}{ Sites } & \multirow{2}{*}{ Villages } & \multirow{2}{*}{$\begin{array}{l}\text { Depths } \\
(\mathrm{cm})\end{array}$} & \multirow{2}{*}{$\begin{array}{c}\mathrm{pH} \\
\text { (eau) }\end{array}$} & \multirow{2}{*}{$\begin{array}{l}\text { C-org } \\
(\mathrm{g} / \mathrm{kg})\end{array}$} & \multirow{2}{*}{$\begin{array}{c}\text { N-total } \\
(\mathrm{g} / \mathrm{kg})\end{array}$} & \multirow{2}{*}{$\mathrm{C} / \mathrm{N}$} & \multirow{2}{*}{$\begin{array}{l}\text { P-Brayl } \\
(\mathrm{mg} / \mathrm{kg})\end{array}$} & \multicolumn{3}{|c|}{ B.E $(\mathrm{cmol} / \mathrm{kg})$} \\
\hline & & & & & & & & $\mathrm{Ca}^{2+}$ & $\mathrm{Mg}^{2+}$ & $\mathrm{K}^{+}$ \\
\hline Dassa & Miniffi & $0-20$ & 7.8 & 8.0 & 0.6 & 13.3 & 47.5 & 33.3 & 2.3 & 2.2 \\
\hline Allada & Niaouli & $0-20$ & 5.9 & 10.6 & 0.7 & 14.5 & 35.5 & 6.1 & 4.2 & 0.7 \\
\hline
\end{tabular}

C-org: Organic Carbon; N-total: total nitrogen; P-Brayl: Assimilable phosphorus; B.E: Exchangeable Bases.

biostimulant $\mathrm{T}_{15}$ : peat $+P$. syringae. It induced an increase of $83.06 \%$ compared to the control formulation ( $\mathrm{T}_{12}$ : peat without PGPR). A highly significant difference $(p<0.001)$ existed between treatments at 31 DAS.

\subsection{Evolution of the Height of Maize Plants on Ferruginous Soil}

Figure 3 illustrates the evolution over time of the height of maize plants on ferruginous soil. The advantage of applying biofertilizer on this soil was most noticeable with $\mathrm{T}_{11}$ : clay-mud $+S$. marcescens. This treatment showed an improvement of $89.72 \%$ compared to the control ( $\mathrm{T}_{6}$ : clay-mud without PGPR). There was a highly significant difference between the treatments $(p<0.001)$ at 


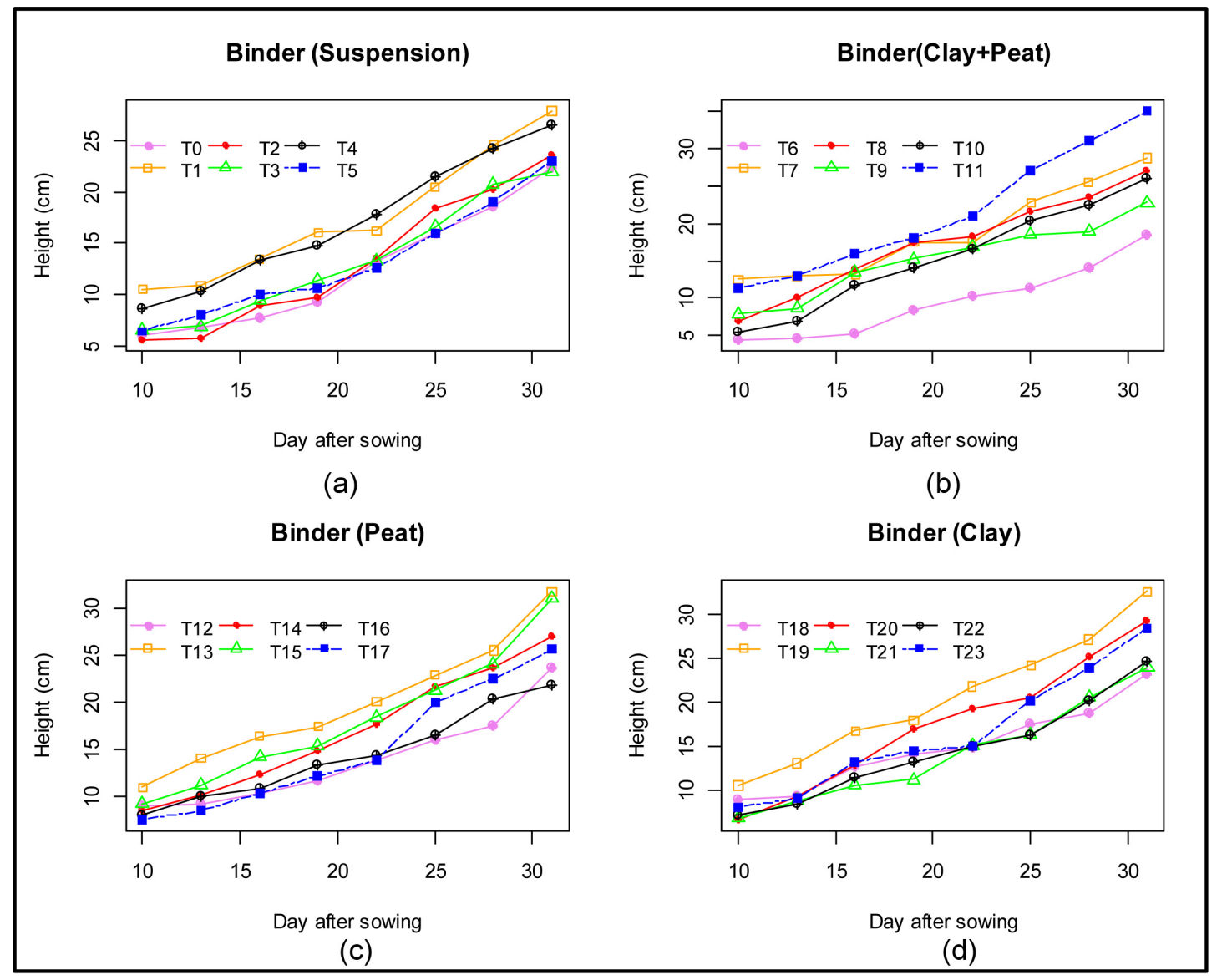

Figure 3. Effect of biostimulants on the height of maize plants on ferruginoussoil. (a) Suspension, (b) Biostimulant based on clay-peat, (c) Biostimulant based on peat, (d) Biostimulant based on clay. $\mathrm{T}_{0}$ : control, $\mathrm{T}_{1}:$ P. putida $\mathrm{S}, \mathrm{T}_{2}:$ P. putida $\mathrm{N}, \mathrm{T}_{3}:$ P. syringae, $\mathrm{T}_{4}:$ B. thuringiensis, $\mathrm{T}_{5}: S$. marcescens, $\mathrm{T}_{6}$ : clay-peat (without $\mathrm{PGPR}$ ), $\mathrm{T}_{7}$ : clay-peat $+P$. putida $\mathrm{S}, \mathrm{T}_{8}:$ clay-peat $+P$. putida $\mathrm{N}$, $\mathrm{T}_{9}$ : clay-peat + P. syringae, $\mathrm{T}_{10}$ : clay-peat + B. thuringiensis, $\mathrm{T}_{11}$ : peat-clay $+S$. marcescens, $\mathrm{T}_{12}:$ peat (without $\mathrm{PGPR}$ ), $\mathrm{T}_{13}: \mathrm{peat}+P$. putida $\mathrm{S}, \mathrm{T}_{14}$ : peat + P. putida $\mathrm{N}, \mathrm{T}_{15}$ : peat + P. syringae, $\mathrm{T}_{16}:$ peat $+B$. thuringiensis, $\mathrm{T}_{17}:$ peat $+S$. marcescens, $\mathrm{T}_{18}$ : clay (without PGPR), $\mathrm{T}_{19}$ : clay + P. putida $\mathrm{S}, \mathrm{T}_{20}$ : clay + P. putida $\mathrm{N}, \mathrm{T}_{21}:$ clay + P. syringae, $\mathrm{T}_{22}:$ clay + B. thuringiensis, $\mathrm{T}_{23}:$ clay $+S$. marcescens.

\subsection{Evolution of the Stem Diameter of Maize Plants on Ferrallitic Soil}

The curves in Figure 4 illustrated the evolution over time of stem diameter of maize plants on ferrallitic soil. The best diameter was recorded with the biostimulant $\mathrm{T}_{15}$ : peat $+P$. syringae. This biostimulant had an increase of $44.57 \%$ compared to the control $\left(\mathrm{T}_{12}\right.$ : peat without PGPR). A highly significant difference $(p<0.001)$ existed between treatments at 31 DAS.

\subsection{Evolution of the Stem Diameter of Maize Plants on Ferruginous Soil}

The curves in Figure 5 illustrate the evolution over time of stem diameter of maize plants on ferruginous soil. The best stem diameter was recorded with the solid biostimulant $\mathrm{T}_{10}$ : peat-clay $+B$. thuringiensis with an overrun of $66.27 \%$ of the control. There was a highly significant difference $(p<0.001)$ between the different treatments at 31 DAS. 


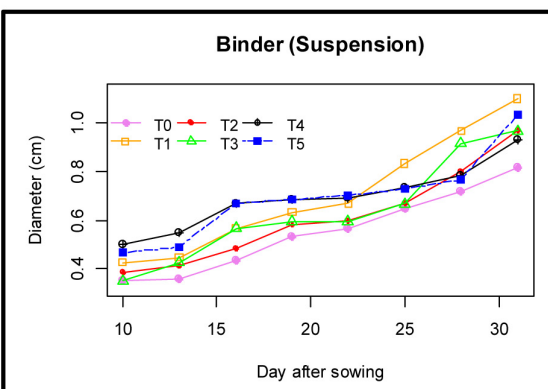

(a)

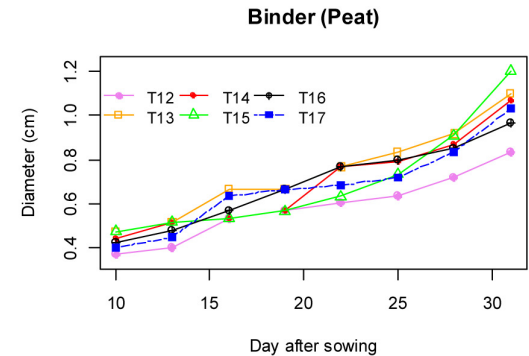

(c)

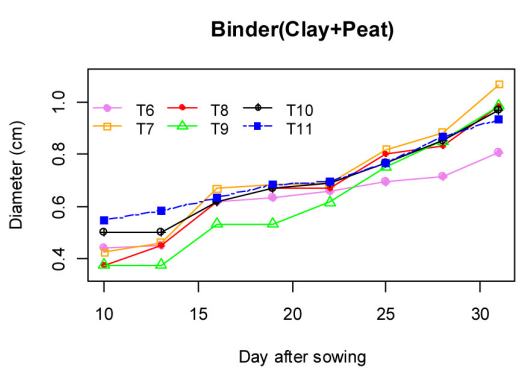

(b)

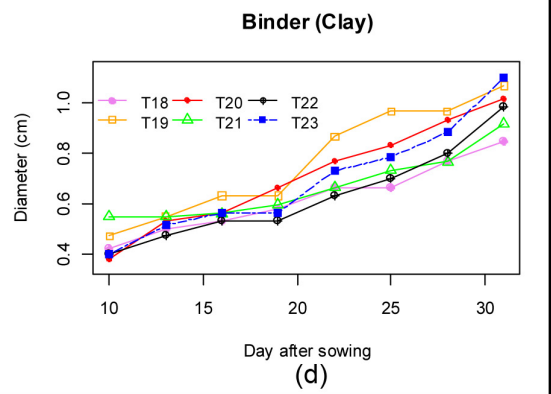

Figure 4. Effect of biostimulants on the stem diameter of maize plants on ferrallitic soil. (a) Suspension, (b) Biostimulant based on clay-peat, (c) Biostimulant based on peat, (d) Biostimulant based on clay. $\mathrm{T}_{0}:$ control, $\mathrm{T}_{1}:$ P. putida S, $\mathrm{T}_{2}:$ P. putida N, $\mathrm{T}_{3}:$ P. syringae, $\mathrm{T}_{4}:$ B. thuringiensis, $\mathrm{T}_{5}: S$. marcescens, $\mathrm{T}_{6}$ : clay-peat (without $\mathrm{PGPR}$ ), $\mathrm{T}_{7}$ : clay-peat $+P$. putida $\mathrm{S}, \mathrm{T}_{8}:$ clay-peat $+P$. putida $\mathrm{N}$, $\mathrm{T}_{9}$ : clay-peat + P. syringae, $\mathrm{T}_{10}$ : clay-peat + B. thuringiensis, $\mathrm{T}_{11}$ : peat-clay $+S$. marcescens, $\mathrm{T}_{12}:$ peat (without PGPR), $\mathrm{T}_{13}:$ peat $+P$. putida $\mathrm{S}, \mathrm{T}_{14}$ : peat $+P$. putida $\mathrm{N}, \mathrm{T}_{15}$ : peat + P. syringae, $\mathrm{T}_{16}$ : peat $+B$. thuringiensis, $\mathrm{T}_{17}:$ peat $+S$. marcescens, $\mathrm{T}_{18}:$ clay (without $\mathrm{PGPR}), \mathrm{T}_{19}:$ clay $+P$. putida $\mathrm{S}, \mathrm{T}_{20}:$ clay + P. putida $\mathrm{N}, \mathrm{T}_{21}:$ clay + P. syringae, $\mathrm{T}_{22}:$ clay $+B$. thuringiensis, $\mathrm{T}_{23}:$ clay $+S$. marcescens.

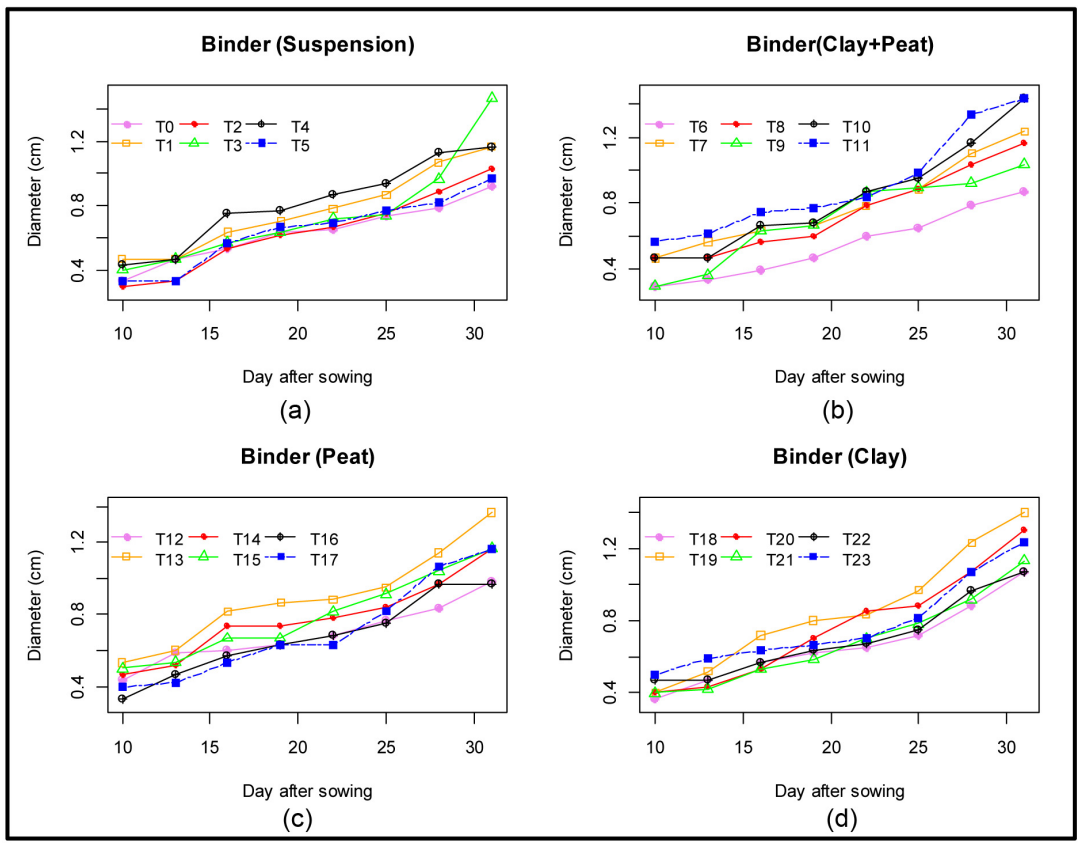

Figure 5. Effect of biostimulants on the stem diameter of maize plants on ferruginous soil. (a) Suspension, (b) Biostimulant based on clay-peat, (c) Biostimulant based on peat, (d) Biostimulant based on clay. $\mathrm{T}_{0}:$ control, $\mathrm{T}_{1}:$ P. putida $\mathrm{S}, \mathrm{T}_{2}: P$. putida $\mathrm{N}, \mathrm{T}_{3}: P$. syringae, $\mathrm{T}_{4}:$ B. thuringiensis, $\mathrm{T}_{5}: S$. marcescens, $\mathrm{T}_{6}$ : clay-peat (without $\mathrm{PGPR}$ ), $\mathrm{T}_{7}$ : clay-peat $+P$. putida $\mathrm{S}, \mathrm{T}_{8}:$ clay-peat $+P \cdot$ putida $\mathrm{N}, \mathrm{T}_{9}$ : clay-peat $+P$. syringae, $\mathrm{T}_{10}$ : clay-peat $+B$. thuringiensis, $\mathrm{T}_{11}:$ peat-clay $+S$. marcescens, $\mathrm{T}_{12}:$ peat (without $\mathrm{PGPR}$ ), $\mathrm{T}_{13}:$ peat + P. putida $\mathrm{S}, \mathrm{T}_{14}:$ peat + P. putida $\mathrm{N}, \mathrm{T}_{15}$ : peat + P. syringae, $\mathrm{T}_{16}:$ peat + B. thuringiensis, $\mathrm{T}_{17}:$ peat $+S$. marcescens, $\mathrm{T}_{18}:$ clay (without $\mathrm{PGPR}), \mathrm{T}_{19}$ : clay + P. putida $\mathrm{S}, \mathrm{T}_{20}$ : clay + P. putida $\mathrm{N}, \mathrm{T}_{21}:$ clay + P. syringae, $\mathrm{T}_{22}:$ clay $+B$. thuringiensis, $\mathrm{T}_{23}:$ clay $+S$. marcescens. 


\subsection{Leaf Area, Aerial Biomass and Underground Biomass on Ferrallitic Soil}

Table 2 provides data on leaf area, aerial biomass and underground biomass on ferrallitic soils. The best leaf area $\left(154.68 \mathrm{~cm}^{2}\right)$ was recorded with the application of the biostimulant $\mathrm{T}_{19}$ : clay $+P$. putida $\mathrm{S}$ which had an increase of $66.10 \%$ compared to the control formulation ( $\mathrm{T}_{18}$ : clay without PGPR). The highest aerial biomass was recorded with the application of biostimulant $\mathrm{T}_{19}$ : clay $+P$. putida $\mathrm{S}$ which exceeded the control formulation ( $\mathrm{T}_{18}$ : clay without PGPR) by $63.49 \%$. The highest underground biomass was obtained with the biostimulant $\mathrm{T}_{19}$ : clay $+P$. putida S with an increase of $53.98 \%$ compared to the control $\left(\mathrm{T}_{18}\right.$ : clay without PGPR). A highly significant difference $(p<0.001)$ existed between the different treatments at 31 DAS for all parameters.

\subsection{Leaf Area, Aerial Biomass and Underground Biomass on Ferruginous Soil}

Table 3 provides data on leaf area, aerial biomass and underground biomass on ferruginous soils. The biostimulant formulated with the combination of peat-clay $+S$. marcescens $\left(\mathrm{T}_{11}\right)$ gave the best leaf area $\left(188.28 \mathrm{~cm}^{2}\right)$ with an excess of $112.36 \%$ compared to the control formulation $\left(\mathrm{T}_{6}\right.$ : peat-clay without PGPR). The highest value of aerial dry biomass was recorded with the application of the biostimulant $\mathrm{T}_{19}$ : clay $+P$. putida $\mathrm{S}$ which exceeded the control formulation ( $\mathrm{T}_{18}$ : clay without PGPR) by $97.12 \%$. On ferruginous soil, it is with the biostimulant $\mathrm{T}_{15}$ : peat $+P$. syringae that we have a better underground biomass of maize plants. This formulation exceeded the control ( $\mathrm{T}_{12}:$ peat without PGPR) by $42.68 \%$. However, a highly significant difference existed between the different treatments at 31 DAS $(p<0.001)$ for all parameters.

\subsection{Correlation between Growth and Yield of Maize Plants on Ferrallitic Soils}

Principal Component Analysis (PCA) on the different maize plant growth and yield parameters showed that the first two axes retain $85.99 \%$ of the total variance. The projection of individuals indicated three groups of treatments (Figure 6). The first group $\left(G_{1}\right)$ consisted of the four controls $T_{0}, T_{6}, T_{12}$ and $T_{18}$. The plants maintained under these treatments were characterised by an average height of $15.3 \mathrm{~cm} \pm 1.06$; an average stem diameter of $0.83 \mathrm{~cm} \pm 0.02$; an average leaf area of $86.25 \mathrm{~cm}^{2} \pm 8.32$; an average aerial biomass of $27.07 \mathrm{~g} \pm 1.35$ and an average underground biomass of $16.45 \mathrm{~g} \pm 0.4$. The second group $\left(\mathrm{G}_{2}\right)$ consisted of 10 treatments $\mathrm{T}_{2}, \mathrm{~T}_{3}, \mathrm{~T}_{4}, \mathrm{~T}_{5}, \mathrm{~T}_{10}, \mathrm{~T}_{14}, \mathrm{~T}_{16}, \mathrm{~T}_{17}, \mathrm{~T}_{21}$ and $\mathrm{T}_{22}$. The plants that benefited from the treatments of this group G2, had an average height of $19.58 \mathrm{~cm} \pm$ 1.3; an average stem diameter of $0.98 \mathrm{~cm} \pm 0.05$; an average leaf area of 106.64 $\mathrm{cm}^{2} \pm 11.99$; an average aerial biomass of $29.05 \mathrm{~g} \pm 2.75$ and an average underground biomass of $19.86 \mathrm{~g} \pm 2.36$. The third group $\left(\mathrm{G}_{3}\right)$ consisted of 10 treatments $\mathrm{T}_{1}, \mathrm{~T}_{7}, \mathrm{~T}_{8}, \mathrm{~T}_{9}, \mathrm{~T}_{11}, \mathrm{~T}_{13}, \mathrm{~T}_{15}, \mathrm{~T}_{19}, \mathrm{~T}_{20}$ and $\mathrm{T}_{23}$. The plants subjected to these 
Table 2. Leaf area, aerial biomass and underground biomasson ferrallitic soils.

\begin{tabular}{|c|c|c|c|c|c|}
\hline \multirow[t]{2}{*}{ Treatment } & Leaf surface $\left(\mathrm{cm}^{2}\right)$ & \multirow[t]{2}{*}{ Treatment } & Aerial Biomass (g) & \multirow[t]{2}{*}{ Treatment } & $\begin{array}{l}\text { Underground } \\
\text { biomass (g) }\end{array}$ \\
\hline & Mean \pm sd & & Mean \pm sd & & Mean \pm sd \\
\hline T19 & $154.68 \pm 3.97^{\mathrm{a}}$ & T19 & $45.32 \pm 0.76^{\mathrm{a}}$ & T19 & $25.50 \pm 1.99^{\mathrm{a}}$ \\
\hline T8 & $152.47 \pm 6.23^{\mathrm{a}}$ & T7 & $36.40 \pm 0.52^{\mathrm{b}}$ & T17 & $23.65 \pm 1.05^{\mathrm{b}}$ \\
\hline T7 & $147.73 \pm 4.46^{\mathrm{ab}}$ & $\mathrm{T} 13$ & $35.32 \pm 0.33^{b c}$ & $\mathrm{~T} 16$ & $16.64 \pm 0.70^{\mathrm{gh}}$ \\
\hline T15 & $137.56 \pm 1.15^{\mathrm{bc}}$ & $\mathrm{T} 11$ & $34.72 \pm 0.35^{\mathrm{bcd}}$ & T20 & $21.37 \pm 0.96^{c}$ \\
\hline $\mathrm{T} 20$ & $137.17 \pm 23.50^{\mathrm{bc}}$ & $\mathrm{T} 8$ & $34.17 \pm 0.45^{\mathrm{bcd}}$ & $\mathrm{T} 7$ & $21.28 \pm 0.43^{c}$ \\
\hline $\mathrm{T} 13$ & $128.25 \pm 4.34^{\mathrm{cd}}$ & $\mathrm{T} 14$ & $33.35 \pm 0.85^{\mathrm{cde}}$ & $\mathrm{T} 3$ & $21.08 \pm 0.04^{c}$ \\
\hline $\mathrm{T} 1$ & $127.17 \pm 5.88^{\mathrm{cd}}$ & $\mathrm{T} 17$ & $32.80 \pm 0.33^{\mathrm{def}}$ & $\mathrm{T} 13$ & $20.87 \pm 2.52^{c}$ \\
\hline $\mathrm{T} 23$ & $125.05 \pm 6.08^{\mathrm{cd}}$ & $\mathrm{T} 16$ & $32.34 \pm 0.66^{\mathrm{def}}$ & $\mathrm{T} 22$ & $20.63 \pm 0.43^{\mathrm{cd}}$ \\
\hline $\mathrm{T} 11$ & $124.94 \pm 5.58^{\mathrm{cd}}$ & T9 & $32.34 \pm 0.66^{\mathrm{def}}$ & $\mathrm{T} 23$ & $19.81 \pm 0.64^{\text {cde }}$ \\
\hline $\mathrm{T} 3$ & $124.43 \pm 2.03^{\mathrm{cd}}$ & $\mathrm{T} 23$ & $31.57 \pm 1.81^{\mathrm{ef}}$ & $\mathrm{T} 21$ & $19.80 \pm 1.32^{\text {cde }}$ \\
\hline T9 & $121.33 \pm 0.39^{\mathrm{cd}}$ & $\mathrm{T} 20$ & $31.04 \pm 0.56^{\mathrm{efg}}$ & $\mathrm{T} 1$ & $19.58 \pm 1.01^{\mathrm{cdef}}$ \\
\hline T5 & $118.10 \pm 10.00^{\mathrm{de}}$ & $\mathrm{T} 1$ & $30.50 \pm 1.12^{\mathrm{fgh}}$ & T8 & $19.23 \pm 0.53^{\text {cdefg }}$ \\
\hline $\mathrm{T} 21$ & $115.90 \pm 0.13^{\mathrm{de}}$ & $\mathrm{T} 21$ & $29.04 \pm 1.32^{\mathrm{ghi}}$ & $\mathrm{T} 11$ & $18.94 \pm 0.28^{\text {cdefg }}$ \\
\hline $\mathrm{T} 22$ & $113.98 \pm 1.80^{\mathrm{de}}$ & T6 & $28.60 \pm 2.01^{\mathrm{hi}}$ & $\mathrm{T} 2$ & $18.68 \pm 0.39^{\text {cdefgh }}$ \\
\hline $\mathrm{T} 2$ & $112.37 \pm 7.74^{\mathrm{def}}$ & T22 & $28.38 \pm 1.74^{\mathrm{hi}}$ & T5 & $18.62 \pm 0.10^{\text {cdefgh }}$ \\
\hline $\mathrm{T} 17$ & $104.92 \pm 0.28^{\mathrm{efg}}$ & $\mathrm{T} 2$ & $27.94 \pm 1.01^{\mathrm{ij}}$ & $\mathrm{T} 14$ & $18.04 \pm 2.01^{\operatorname{defgh}}$ \\
\hline T14 & $99.40 \pm 0.88^{\text {fgh }}$ & T18 & $27.72 \pm 1.32^{\mathrm{ijk}}$ & $\mathrm{T} 4$ & $17.94 \pm 1.29^{\operatorname{defgh}}$ \\
\hline $\mathrm{T} 18$ & $95.53 \pm 3.22^{\mathrm{gh}}$ & T5 & $27.06 \pm 0.66^{\mathrm{ijk}}$ & $\mathrm{T} 15$ & $17.64 \pm 0.72^{\text {efgh }}$ \\
\hline $\mathrm{T} 10$ & $94.98 \pm 0.51^{\mathrm{gh}}$ & $\mathrm{T} 10$ & $26.66 \pm 0.27^{\mathrm{ijk}}$ & T12 & $16.95 \pm 0.17^{\mathrm{fgh}}$ \\
\hline T16 & $92.50 \pm 5.07^{\mathrm{gh}}$ & $\mathrm{T} 4$ & $26.52 \pm 1.33^{\mathrm{ijk}}$ & $\mathrm{T} 10$ & $16.64 \pm 0.70^{g h}$ \\
\hline $\mathrm{T} 4$ & $89.81 \pm 7.09^{\mathrm{gh}}$ & $\mathrm{T} 12$ & $26.40 \pm 1.32^{\mathrm{ijk}}$ & T9 & $16.60 \pm 0.68^{\mathrm{gh}}$ \\
\hline $\mathrm{T} 12$ & $87.45 \pm 0.60^{\mathrm{hi}}$ & $\mathrm{T} 3$ & $26.39 \pm 0.17^{\mathrm{ijk}}$ & $\mathrm{T} 18$ & $16.56 \pm 0.53^{\mathrm{gh}}$ \\
\hline T6 & $86.72 \pm 5.04^{\mathrm{hi}}$ & T0 & $25.57 \pm 1.57^{\mathrm{jk}}$ & T6 & $16.16 \pm 0.32^{\mathrm{h}}$ \\
\hline T0 & $75.29 \pm 5.64^{\mathrm{i}}$ & $\mathrm{T} 15$ & $25.03 \pm 0.05^{\mathrm{k}}$ & T0 & $16.10 \pm 0.35^{\mathrm{h}}$ \\
\hline $\mathrm{DF}$ & 23 & $\mathrm{DF}$ & 23 & $\mathrm{DF}$ & 23 \\
\hline$F$ value & 32.78 & F value & 58.28 & $F$ value & 18.71 \\
\hline $\operatorname{Pr}(>\mathrm{F})$ & $<0.001^{\star * *}$ & $\operatorname{Pr}(>\mathrm{F})$ & $<0.001^{\star * *}$ & $\operatorname{Pr}(>\mathrm{F})$ & $<0.001^{\star * *}$ \\
\hline
\end{tabular}

$\mathrm{T}_{0}$ : control, $\mathrm{T}_{1}:$ P. putida $\mathrm{S}, \mathrm{T}_{2}:$ P. putida $\mathrm{N}, \mathrm{T}_{3}:$ P. syringae, $\mathrm{T}_{4}:$ B. thuringiensis, $\mathrm{T}_{5}:$ S. marcescens, $\mathrm{T}_{6}$ : clay-peat (without PGPR), $\mathrm{T}_{7}$ : clay-peat $+P$. putida $\mathrm{S}, \mathrm{T}_{8}$ : clay-peat + P. putida $\mathrm{N}, \mathrm{T}_{9}$ : clay-peat + P. syringae, $\mathrm{T}_{10}$ : clay-peat + B. thuringiensis, $\mathrm{T}_{11}$ : peat-clay $+S$. marcescens, $\mathrm{T}_{12}$ : peat (without PGPR), $\mathrm{T}_{13}:$ peat $+P$. putida $\mathrm{S}, \mathrm{T}_{14}$ : peat + P. putida $\mathrm{N}, \mathrm{T}_{15}$ : peat + P. syringae, $\mathrm{T}_{16}:$ peat + B. thuringiensis, $\mathrm{T}_{17}$ : peat $+S$. marcescens, $\mathrm{T}_{18}$ : clay (without PGPR), $\mathrm{T}_{19}$ : clay $+P$. putida $\mathrm{S}, \mathrm{T}_{20}$ : clay $+P$. putida $\mathrm{N}, \mathrm{T}_{21}$ : clay $+P$. syringae, $\mathrm{T}_{22}$ : clay $+B$. thuringiensis, $\mathrm{T}_{23}$ : clay $+S$. marcescens. 
Table 3. Leaf area, aerial biomass and underground biomasson ferruginous soils.

\begin{tabular}{|c|c|c|c|c|c|}
\hline \multirow[t]{2}{*}{ Treatment } & Leaf surface $\left(\mathrm{cm}^{2}\right)$ & \multirow[t]{2}{*}{ Treatment } & Aerial Biomass (g) & \multirow[t]{2}{*}{ Treatment } & $\begin{array}{l}\text { Underground } \\
\text { biomass (g) }\end{array}$ \\
\hline & Mean \pm sd & & Mean \pm sd & & Mean \pm sd \\
\hline $\mathrm{T} 11$ & $188.28 \pm 3.97^{\mathrm{a}}$ & T19 & $52.79 \pm 2.05^{\mathrm{a}}$ & T15 & $21.26 \pm 0.47^{\mathrm{a}}$ \\
\hline $\mathrm{T} 10$ & $181.28 \pm 1.91^{\mathrm{ab}}$ & $\mathrm{T} 13$ & $50.28 \pm 6.27^{\mathrm{ab}}$ & $\mathrm{T} 11$ & $19.48 \pm 0.81^{\mathrm{b}}$ \\
\hline $\mathrm{T} 15$ & $173.25 \pm 2.78^{\mathrm{bc}}$ & $\mathrm{T} 15$ & $49.01 \pm 0.87^{\mathrm{ab}}$ & T20 & $19.16 \pm 1.14^{\mathrm{bc}}$ \\
\hline T19 & $170.35 \pm 5.36^{\mathrm{bcd}}$ & T11 & $46.50 \pm 0.66^{\mathrm{b}}$ & T10 & $19.00 \pm 0.91^{\mathrm{bc}}$ \\
\hline $\mathrm{T} 7$ & $165.22 \pm 5.11^{\mathrm{cd}}$ & $\mathrm{T} 20$ & $39.23 \pm 1.14^{\mathrm{c}}$ & $\mathrm{T} 19$ & $18.84 \pm 1.37^{\mathrm{bcd}}$ \\
\hline $\mathrm{T} 23$ & $163.31 \pm 10.07^{\mathrm{cd}}$ & $\mathrm{T} 14$ & $39.18 \pm 3.01^{\mathrm{c}}$ & $\mathrm{T} 4$ & $18.51 \pm 0.99^{\mathrm{bcd}}$ \\
\hline $\mathrm{T} 8$ & $162.55 \pm 9.47^{\mathrm{cd}}$ & T10 & $39.01 \pm 2.63^{\mathrm{c}}$ & $\mathrm{T} 13$ & $18.39 \pm 0.77^{\mathrm{bcd}}$ \\
\hline $\mathrm{T} 13$ & $155.82 \pm 3.11^{\mathrm{de}}$ & $\mathrm{T} 4$ & $38.94 \pm 1.72^{\mathrm{c}}$ & $\mathrm{T} 8$ & $17.88 \pm 1.02^{\text {bcde }}$ \\
\hline $\mathrm{T} 20$ & $154.84 \pm 7.57^{\mathrm{de}}$ & $\mathrm{T} 17$ & $37.21 \pm 1.05^{\mathrm{cd}}$ & T7 & $17.80 \pm 0.91^{\text {bcdef }}$ \\
\hline $\mathrm{T} 4$ & $154.28 \pm 1.88^{\mathrm{de}}$ & $\mathrm{T} 23$ & $36.68 \pm 3.45^{\mathrm{cd}}$ & $\mathrm{T} 1$ & $17.42 \pm 0.57^{\text {bcdefg }}$ \\
\hline T16 & $153.60 \pm 5.53^{\mathrm{de}}$ & $\mathrm{T} 7$ & $35.17 \pm 2.84^{\mathrm{cd}}$ & $\mathrm{T} 14$ & $17.40 \pm 0.60^{\text {bcdefg }}$ \\
\hline $\mathrm{T} 1$ & $145.64 \pm 10.52^{\mathrm{ef}}$ & $\mathrm{T} 8$ & $32.48 \pm 1.32^{\mathrm{de}}$ & $\mathrm{T} 17$ & $16.80 \pm 1.20^{\text {bcdefgh }}$ \\
\hline $\mathrm{T} 2$ & $136.50 \pm 9.87^{\mathrm{fg}}$ & $\mathrm{T} 1$ & $30.57 \pm 0.63^{\text {ef }}$ & $\mathrm{T} 23$ & $16.80 \pm 1.20^{\text {bcdefgh }}$ \\
\hline T14 & $130.30 \pm 8.47^{g}$ & T22 & $29.80 \pm 0.91^{\mathrm{ef}}$ & T22 & $16.44 \pm 1.76^{\text {bcdefgh }}$ \\
\hline $\mathrm{T} 17$ & $124.48 \pm 11.20^{\mathrm{gh}}$ & T16 & $27.99 \pm 2.84^{\mathrm{efg}}$ & $\mathrm{T} 2$ & $16.37 \pm 1.26^{\text {cdefghi }}$ \\
\hline T5 & $123.22 \pm 1.24^{\mathrm{gh}}$ & T5 & $27.28 \pm 2.77^{\mathrm{efg}}$ & $\mathrm{T} 3$ & $15.89 \pm 2.61^{\text {defghi }}$ \\
\hline T9 & $114.01 \pm 0.11^{\mathrm{h}}$ & $\mathrm{T} 3$ & $27.06 \pm 2.89^{\mathrm{efg}}$ & T16 & $15.00 \pm 0.60^{\text {efghij }}$ \\
\hline $\mathrm{T} 21$ & $100.96 \pm 6.73^{\mathrm{i}}$ & $\mathrm{T} 18$ & $26.78 \pm 1.41^{\mathrm{efg}}$ & $\mathrm{T} 12$ & $14.90 \pm 0.14^{\text {fghij }}$ \\
\hline $\mathrm{T} 18$ & $96.04 \pm 0.21^{\mathrm{ij}}$ & $\mathrm{T} 2$ & $26.60 \pm 0.91^{\mathrm{efg}}$ & T5 & $14.78 \pm 0.70^{\text {ghij }}$ \\
\hline $\mathrm{T} 22$ & $95.63 \pm 2.07^{\mathrm{ij}}$ & $\mathrm{T} 12$ & $26.23 \pm 1.47^{\mathrm{fg}}$ & T9 & $14.60 \pm 1.24^{\text {ghij }}$ \\
\hline $\mathrm{T} 3$ & $92.59 \pm 5.43^{\mathrm{ij}}$ & $\mathrm{T} 21$ & $26.00 \pm 1.83^{\mathrm{fg}}$ & T0 & $14.00 \pm 0.69^{h i j}$ \\
\hline T6 & $88.66 \pm 6.79^{\mathrm{ij}}$ & T6 & $25.20 \pm 1.58^{\mathrm{fg}}$ & $\mathrm{T} 21$ & $13.97 \pm 0.37^{\mathrm{hij}}$ \\
\hline $\mathrm{T} 12$ & $85.37 \pm 10.41^{\mathrm{ij}}$ & T9 & $25.20 \pm 1.20^{\mathrm{fg}}$ & T6 & $13.60 \pm 0.69^{\mathrm{ij}}$ \\
\hline T0 & $82.55 \pm 1.06^{j}$ & T0 & $22.60 \pm 0.92^{\mathrm{g}}$ & T18 & $13.36 \pm 0.96^{\mathrm{j}}$ \\
\hline DF & 23 & DF & 23 & DF & 23 \\
\hline F value & 79.88 & F value & 44.5 & F value & 11.72 \\
\hline $\operatorname{Pr}(>\mathrm{F})$ & $<0.001^{* * *}$ & $\operatorname{Pr}(>\mathrm{F})$ & $<0.001^{* * *}$ & $\operatorname{Pr}(>\mathrm{F})$ & $<0.001^{* * *}$ \\
\hline
\end{tabular}

$\mathrm{T}_{0}$ : control, $\mathrm{T}_{1}:$ P. putida $\mathrm{S}, \mathrm{T}_{2}: P$. putida $\mathrm{N}, \mathrm{T}_{3}:$ P. syringae, $\mathrm{T}_{4}:$ B. thuringiensis, $\mathrm{T}_{5}: S$. marcescens, $\mathrm{T}_{6}$ : clay-peat (without $\mathrm{PGPR}), \mathrm{T}_{7}$ : clay-peat $+P$. putida $\mathrm{S}, \mathrm{T}_{8}$ : clay-peat $+P$. putida $\mathrm{N}, \mathrm{T}_{9}$ : clay-peat $+P$. syringae, $\mathrm{T}_{10}$ : clay-peat $+B$. thuringiensis, $\mathrm{T}_{11}$ : peat-clay $+S$. marcescens, $\mathrm{T}_{12}:$ peat (without PGPR), $\mathrm{T}_{13}:$ peat $+P$. putida $\mathrm{S}, \mathrm{T}_{14}:$ peat + P. putida $\mathrm{N}, \mathrm{T}_{15}:$ peat + P. syringae, $\mathrm{T}_{16}:$ peat + B. thuringiensis, $\mathrm{T}_{17}:$ peat + S. marcescens, $\mathrm{T}_{18}$ : clay (without PGPR), $\mathrm{T}_{19}$ : clay + P. putida $\mathrm{S}, \mathrm{T}_{20}$ : clay + P. putida $\mathrm{N}, \mathrm{T}_{21}$ : clay + P. syringae, $\mathrm{T}_{22}$ : clay + B. thuringiensis, $\mathrm{T}_{23}$ : clay + S. marcescens. 


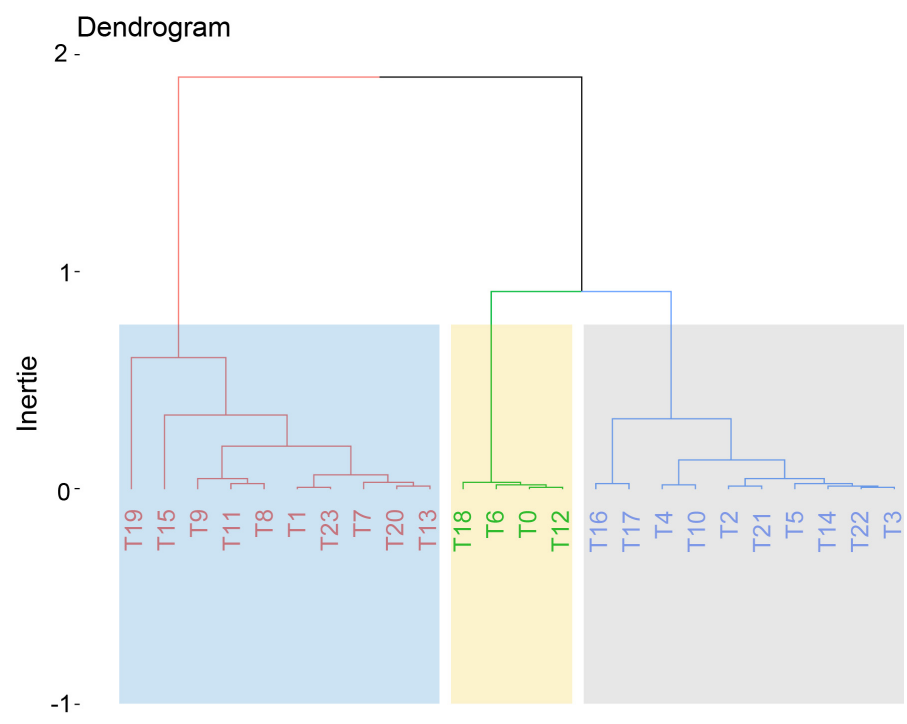

(a)

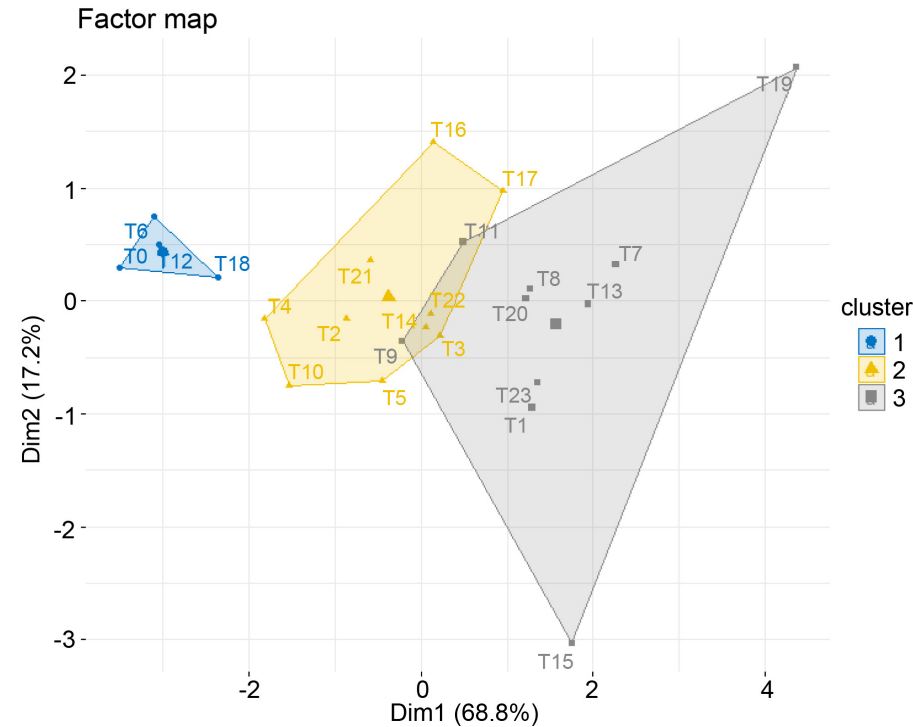

(b)

Figure 6. (a) Dendrogram of treatment groups; (b) Projection in the two dimensions of PCA on ferrallitic soils. $\mathrm{T}_{0}$ : control, $\mathrm{T}_{1}: P$. putida $\mathrm{S}, \mathrm{T}_{2}: P$. putida $\mathrm{N}, \mathrm{T}_{3}: P$. syringae, $\mathrm{T}_{4}: B$. thuringiensis, $\mathrm{T}_{5}: S$. marcescens, $\mathrm{T}_{6}$ : clay-peat (without $\mathrm{PGPR}$ ), $\mathrm{T}_{7}$ : clay-peat + P. putida $\mathrm{S}$, $\mathrm{T}_{8}$ : clay-peat $+P$. putida $\mathrm{N}, \mathrm{T}_{9}$ : clay-peat $+P$. syringae, $\mathrm{T}_{10}$ : clay-peat $+B$. thuringiensis, $\mathrm{T}_{11}$ : peat-clay $+S$. marcescens, $\mathrm{T}_{12}$ : peat (without $\mathrm{PGPR}$ ), $\mathrm{T}_{13}:$ peat + P. putida $\mathrm{S}, \mathrm{T}_{14}:$ peat + P. putida $\mathrm{N}, \mathrm{T}_{15}:$ peat + P. syringae, $\mathrm{T}_{16}:$ peat + B. thuringiensis, $\mathrm{T}_{17}:$ peat $+S$. marcescens, $\mathrm{T}_{18}$ : clay (without PGPR), $\mathrm{T}_{19}$ : clay $+P$. putida $\mathrm{S}, \mathrm{T}_{20}$ : clay $+P$. putida $\mathrm{N}, \mathrm{T}_{21}:$ clay + P. syringae, $\mathrm{T}_{22}$ : clay $+B$. thuringiensis, $\mathrm{T}_{23}$ : clay $+S$. marcescens.

treatments had an average height of $22.82 \mathrm{~cm} \pm 1.9$; an average stem diameter of $1.05 \mathrm{~cm} \pm 0.08$; an average leaf area of $135.64 \mathrm{~cm}^{2} \pm 12.28$; an average aerial biomass of $33.64 \mathrm{~g} \pm 5.22$; and an average underground biomass of $20.09 \mathrm{~g} \pm$ 2.44. The $\mathrm{G}_{3}$ group gave the best performance in both growth and yield parameters while the $\mathrm{G}_{1}$ group gave the lowest performance. On ferrallitic soil the biostimulant $\mathrm{T}_{19}$ : clay + P. putida $\mathrm{S}$ is the best of all the others in group $\mathrm{G}_{3}$. 


\subsection{Correlation between Growth and Yield of Maize Plants on Ferruginous Soils}

Principal Component Analysis (PCA) on the different maize plant growth and yield parameters showed that the first two axes retained $89.86 \%$ of the total variance. The projection of individuals indicated three groups of treatments (Figure 7). The first group $\left(G_{1}\right)$ consists of 11 treatments $T_{0}, T_{2}, T_{3}, T_{5}, T_{6}, T_{9}$,

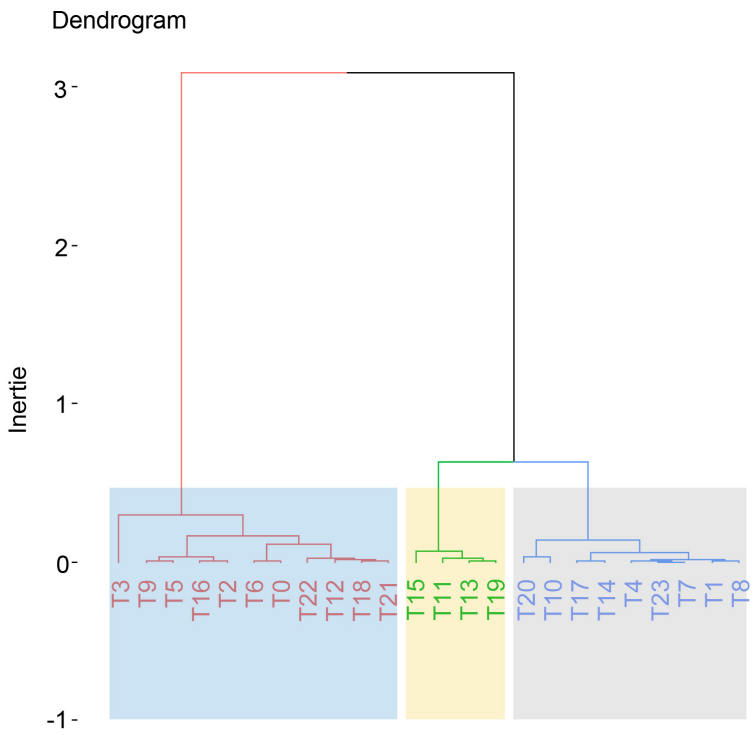

(a)

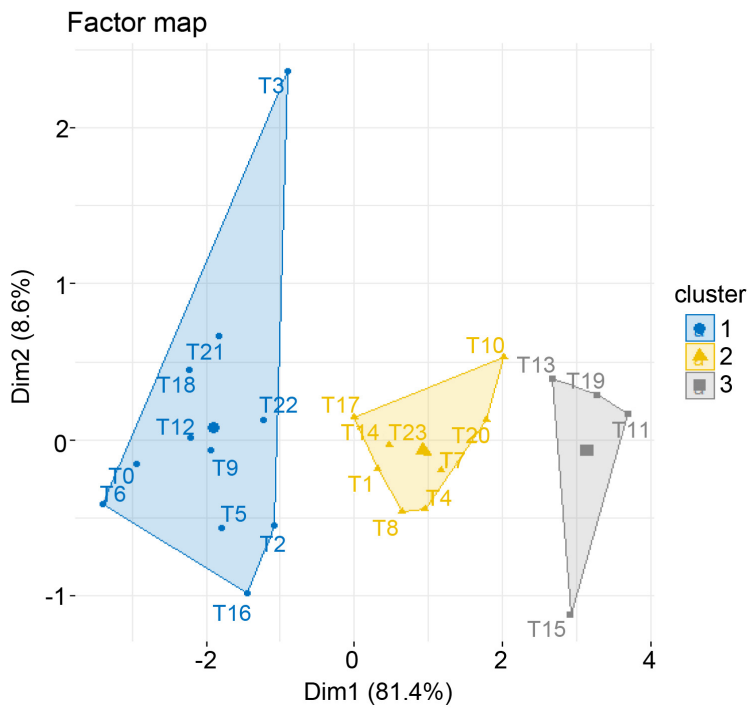

(b)

Figure 7. (a) Dendrogram of treatment groups; (b) Projection in the two dimensions of PCA on ferruginous soils. $\mathrm{T}_{0}$ : control, $\mathrm{T}_{1}:$ P. putida $\mathrm{S}, \mathrm{T}_{2}:$ P. putida $\mathrm{N}, \mathrm{T}_{3}:$ P. syringae, $\mathrm{T}_{4}$ : B. thuringiensis, $\mathrm{T}_{5}: S$. marcescens, $\mathrm{T}_{6}$ : clay-peat (without $\mathrm{PGPR}$ ), $\mathrm{T}_{7}$ : clay-peat + P. putida $\mathrm{S}, \mathrm{T}_{8}$ : clay-peat $+P$. putida $\mathrm{N}, \mathrm{T}_{9}$ : clay-peat $+P$. syringae, $\mathrm{T}_{10}$ : clay-peat $+B$. thuringiensis, $\mathrm{T}_{11}$ : peat-clay $+S$. marcescens, $\mathrm{T}_{12}$ : peat (without $\left.\mathrm{PGPR}\right), \mathrm{T}_{13}$ : peat $+P$. putida $\mathrm{S}, \mathrm{T}_{14}:$ peat $+P$. putida $\mathrm{N}, \mathrm{T}_{15}$ : peat + P. syringae, $\mathrm{T}_{16}$ : peat + B. thuringiensis, $\mathrm{T}_{17}$ : peat $+S$. marcescens, $\mathrm{T}_{18}$ : clay (without $\mathrm{PGPR}$ ), $\mathrm{T}_{19}$ : clay + P. putida $\mathrm{S}, \mathrm{T}_{20}$ : clay + P. putida $\mathrm{N}, \mathrm{T}_{21}$ : clay + $P$. syringae, $\mathrm{T}_{22}$ : clay $+B$. thuringiensis, $\mathrm{T}_{23}$ : clay $+S$. marcescens. 
$\mathrm{T}_{12}, \mathrm{~T}_{16}, \mathrm{~T}_{18}, \mathrm{~T}_{21}$ and $\mathrm{T}_{22}$. The plants maintained under these treatments are characterised by an average height of $22.69 \mathrm{~cm} \pm 1.63$; an average stem diameter of $1.05 \mathrm{~cm} \pm 0.16$; an average leaf area of $106.29 \mathrm{~cm}^{2} \pm 22.90$; an average aerial biomass of $26.43 \mathrm{~g} \pm 1.82$ and an average underground biomass of $14.81 \mathrm{~g} \pm$ 1.06. The second group $\left(G_{2}\right)$ consists of nine treatments $T_{1}, T_{4}, T_{7}, T_{8}, T_{10}, T_{14}$, $\mathrm{T}_{17}, \mathrm{~T}_{20}$ and $\mathrm{T}_{23}$. The plants that have benefited from the treatments in this group $\mathrm{G}_{2}$ have an average height of $27.38 \mathrm{~cm} \pm 1.27$; an average stem diameter of 1.23 $\mathrm{cm} \pm 0.09$; an average leaf area of $153.58 \mathrm{~cm}^{2} \pm 17.86$; an average aerial biomass of $36.50 \mathrm{~g} \pm 3.17$ and an average underground biomass of $17.86 \mathrm{~g} \pm 0.87$. The third group $\left(G_{3}\right)$ consists of four treatments $T_{11}, T_{13}, T_{15}$ and $T_{19}$. The plants subjected to these treatments have an average height of $32.67 \mathrm{~cm} \pm 1.74$; an average stem diameter of $1.34 \mathrm{~cm} \pm 0.12$; an average leaf area of $171.93 \mathrm{~cm}^{2} \pm 13.31$; an average aerial biomass of $49.64 \mathrm{~g} \pm 2.62$ and an average underground biomass of $19.50 \mathrm{~g} \pm 1.26$. Group $\mathrm{G}_{3}$ gives the best performance in both growth and yield parameters while group $\mathrm{G}_{1}$ gives the lowest performance. On ferruginous soil, the biostimulant $\mathrm{T}_{15}$ : peat $+P$. syringaeis the best.

\section{Discussion}

Chemical fertilizers are generally used to provide essential nutrients to plants. However, the high cost, availability and environmental concerns related to chemical fertilizers are real problems for agriculture [6]. The use of microbial biostimulants, including PGPR, to improve sustainable agricultural production is a practice that is becoming more widely accepted in intensive agriculture. PGPR are free-living soil bacteria that colonise plant roots and improve growth and yield when applied to seeds or crops [32]. The aims of the study were to develop solid biostimulants based on five rhizobacteria native to Benin's soils and to evaluate their efficacy on the growth and biomass yield of maize under greenhouse conditions on ferrallitic and ferruginous soils.

The first activity was the validation of the best binders in order to use them for the formulation. The bacterial load was constant in the clay and peat biostimulants after two months of incubation. Similar results were reported by [33] in Tunisia with biostimulants composed of Kaolin or talc-kaolin binder and PGPR $P$. trivialis X33d at room temperature. Clay and peat biostimulants maintained a high bacterial population for five to eight months at $25^{\circ} \mathrm{C}$ [34] [35] [36], explains the survival of the bacteria by the availability of labile carbon and nitrogen and also the $\mathrm{pH}$ of the binders (clay, peat).

Analysis of the results of the initial chemical properties of the test soils shows that ferrallitic soil is slightly acidic while ferruginous soil is alkaline. The ferrallitic soil also showed a high level of assimilable phosphorus compared to the ferruginous soil. In general, both types of soil presented a high $\mathrm{C} / \mathrm{N}$ ratio; the sum of exchangeable bases and the cation exchange capacity are low, which reflects their low fertility, as reported by [15]. Moreover, potassium is globally deficient in relation to calcium and magnesium in both soils. Imbalances existed between calcium, magnesium and phosphorus. These results are in line with 
those reported by [37].

Many researchers have indicated that the use of biostimulants plays a key role in improving soil fertility, crop growth and final yield. Their application improves soil biological activity and reduces the use of chemical fertilizers [38]. Recently, [39] reported that the use of microbial biostimulants can provide an alternative for improved nutrient bioavailability in the soil and therefore good crop productivity. Statistical analysis was highly significant $(p<0.001)$ for growth parameters (height, stem diameter and leaf area). Solid biostimulants (peat + P. syringae $\left(\mathrm{T}_{15}\right)$ and clay + P. putida $\mathrm{S}\left(\mathrm{T}_{19}\right)$ ) proved to be more effective than simple inoculation. Similar performance was reported in the work of [40] in Parkistan. These authors found that the application of peat $+B$. Thuringiensis had significantly improved the growth and yield of beans. [41] Observed a highly significant increase in growth parameters and overproduction of chlorophyll a and $\mathrm{b}$ by maize plants treated with the biostimulant. The larger leaf area, more the plant achieves good photosynthesis, which is favourable to better growth [42] [43]. Our results on growth parameters can be explained by the stimulation of good photosynthesis by the formulated solid biostimulants. In general, formulated solid biostimulants improved maize plant growth in height, stem diameter and leaf area more than bacterial suspensions. This difference is due to the effects of binders and other biostimulant additives [39]. According to different authors [44] [45] [46] the increase in plant growth is due to the production capacity of auxin, gibberellic acid, biological nitrogen fixation and improvement of the root system by rhizobacteria. The rhizobacteria $P$. putida $\mathrm{S}$ and $P$. syringaecontained in these biostimulants are able to solubilize phosphate, produce indole acetic acid [47] [48] and promote better nutrient uptake by plants from the soil [11]; which justifies the results obtained with inoculated plants.

Several authors have demonstrated the efficacy of microbial biostimulants on plant yield parameters [49] [50] [51]. It was recently found by [41] that $A$. brasilense biostimulant HM053 significantly increased maize yield by $53 \%$ compared to the control. In our study, biostimulants improved yield parameters in a highly significant way $(p<0.001)$. The best biomasses were obtained with the application of biostimulants $\mathrm{T}_{15}$ : peat + P. syringae, $\mathrm{T}_{19}$ : clay + P. putida $\mathrm{S}$ on both soil types. These results are in line with those of [52] and [53], who had increases of $33.85 \%$ and $80 \%$ in the biomass of Poaceae by applying biostimulants. Thanks to a better implantation, good water infiltration, a well-fed plant ensures a good yield. The application of biostimulants is one of the appropriate methods to improve phosphorus availability [39] [54] [55]. On these soils, application of the formulated solid biostimulants $\mathrm{T}_{15}$ : peat + P. syringae and $\mathrm{T}_{19}$ : clay + P. putida $\mathrm{S}$ improves maize growth and yield. These increases can be due to better root growth, better nutrient absorption, high capacity of the clay and peat to retain and provide a large surface area for the PGPRs to survive and function [40] [56].

\section{Conclusion}

Microbial biostimulants are very important for crop improvement. They im- 
prove plant growth and yield. The parameters of vegetative growth and biomass yield of maize have been greatly improved under greenhouse conditions on ferrallitic and ferruginous soils in Benin by the biostimulants clay $+P$. putida and peat $+P$. syringae. These results show that these biostimulants can be used as a biological stimulant for environmentally friendly sustainable agriculture, promoting the use of organic methods to increase the productivity of the various speculations in Benin.

\section{Acknowledgements}

The authors would like to thank the national agricultural research institute of Benin (INRAB) for agreeing to finance this project. Special thanks go to the Director General and his deputy.

\section{Conflicts of Interest}

The authors declare that the research was conducted in the absence of any commercial or financial relationships that could be construed as a potential conflict of interest.

\section{References}

[1] PS/CNS-MAÏS (2018) Plan Stratégique 2019-2034 du Centre National de Spécialisation sur le maïs (CNS-MAÏS), 87 P. Dépôt Légal N 10936 du 27/12/2018, Bibliothèque Nationale du Bénin, 4è Trimestre.

[2] Tshiabukole, J.P.K. (2018) Evaluation de la sensibilité aux stress hydriques du maïs (Zea mays L.) cultivé dans la savane du Sud-Ouest de la RD Congo, cas de Mvuazi. Thèse de Doctorat, Faculté des Sciences Agronomiques, Département de Phytotechnie, Université Pédagogique Nationale, RD Congo, 162.

[3] Adjadi, O., Lokossou, C., Azelokonou, O.G., Bankole, C.D., Djinadou, A.K., Ahoyo Adjovi, R.N. and Adjanohoun, A. (2015) Recueil de mets et de boissons à base de maïs consommés au Bénin. Document Technique et d'Informations (DT\&I). CNS-Maïs, INRAB, PPAAO/WAAPP, ProCAD\& MAEP/Bénin. Dépôt légal No 7931 du 04 juin 2015, 2ème Trimestre Bibliothèque Nationale (BN) du Bénin. 156 p.

[4] Saïdou, A., Balogoun, I., Kone, B., Gnangle, C.P. and Aho, N. (2012) Effet d'un système agroforestier à karité (Vitellariaparadoxacfgaertn) sur le sol et le potentiel de production du maïs (Zeamaize) en zone Soudanienne du Bénin. International Journal of Biological and Chemical Sciences, 6, 2066-2082.

https://doi.org/10.4314/ijbcs.v6i5.16

[5] Boominathan, U., Priya, K., Sabarish, R. and Shalini, A. (2020) Screening PGPR for Improving Seed Germination, Seedling Growth and Yield of Maize (Zea mays L.). Studies in Indian Place Names UGC Care Listed Journal, 40, No. 20.

[6] Ojha, S.K., Benjamin, J.C. and Singh, A.K. (2016) Mass Production of Biofertilizer (Pseudomonas fluorescens). International Journal of Agricultural Science and Research (IJASR), 6, 415-418.

[7] Tabassum, B., Khan, A., Tariq, M., Ramzan, M., Iqbal Khan, M.S., Shahid, N. and Aaliya, K. (2017) Bottlenecks in Commercialisation and Future Prospects of PGPR. Applied Soil Ecology, 121, 102-117. https://doi.org/10.1016/j.apsoil.2017.09.030

[8] Diaz, P.A.E., Baron, N.C. and Rigobelo, E.C. (2019) Bacillus spp. as Plant Growth- 
Promoting Bacteria in Cotton under Greenhouse Conditions. Australian Journal of Crop Science, 13, 2003. https://doi.org/10.21475/ajcs.19.13.12.p2003

[9] Bouraoui, F. and Grizzetti, B. (2013) Modelling Mitigation Options to Reduce Diffuse Nitrogen Water Pollution from Agriculture. Science of the Total Environment, 468-469, 1267-1277. https://doi.org/10.1016/j.scitotenv.2013.07.066

[10] Adoko, M.Y., Agbodjato, N.A., Ouikoun, G.C., Amogou, O., Noumavo, P.A., Sina, H., Koda, A.D., Allagbé, M., AhoyoAdjovi, N., Adjanohoun, A. and Baba-Moussa, L. (2020) Inoculation of Pseudomonas putida in Farmer Environment to Improve Growth and Yield: Maize (Zea mays L.) Trial in Sothern, Central and Northern (Benin). IJPSS, 32, 9-21. https://doi.org/10.9734/ijpss/2020/v32i630288

[11] Adjanohoun, A., Noumavo, P.A., Sikirou, R., Allagbé, M., Gotoechan-Hodonou, H., Dossa, K.K., et al. (2012) Effets des rhizobactéries PGPR sur le rendement et les teneurs en macroéléments du maïs sur sol ferralitique non dégradé au Sud-Bénin. International Journal of Biological and Chemical Sciences, 6, 279-288.

https://doi.org/10.4314/ijbcs.v6i1.24

[12] Amogou, O., Dagbénonbakin, G., Agbodjato, A.N., Noumavo, A.P., Salako, K.V., Adoko, Y.M., GlèlèKakaï, R., Adjanohoun, A. and Baba-Moussa, L. (2019) Applying Rhizobacteria on Maize Cultivation in Northern Benin: Effect on Growth and Yield. Agricultural Sciences, 10, 763-782. http://www.scirp.org/journal/as https://doi.org/10.4236/as.2019.106059

[13] Agbodjato, N.A., Noumavo, P.A., Adjanohoun, A., Agbessi, L. and Baba-Moussa, L. (2016) Synergistic Effects of Plant Growth Promoting Rhizobacteria and Chitosan on in Vitro Seeds Germination, Greenhouse Growth, and Nutrient Uptake of Maize (Zea mays L.). Biotechnology Research International, 2016, Article ID: 7830182. http://www.hindawi.com/journals/btri/2016/7830182 https://doi.org/10.1155/2016/7830182

[14] Noumavo, A.P., Kochoni, E., Didagbé, Y.O., Adjanohoun, A., Allagbé, M., Sikirou, R., Gachomo, E.W., Kotchoni, O.S. and Baba-Moussa, L. (2013) Effect of Different Plant Growth Promoting Rhizobacteria on Maize Seed Germination and Seedling Development. American Journal of Plant Sciences, 4, 1013-1021.

https://doi.org/10.4236/ajps.2013.45125

[15] Adjanohoun, A., Baba-Moussa, L., Glèlèkakaï, R., Allagbé, M., Yèhouénou, B., Gotoechan-Hodonou, H., et al. (2011) Caractérisation des rhizobactéries potentiellement promotrices de la croissance végétative du maïs dans différents agrosystèmes $\mathrm{du}$ Sud-Bénin. International Journal of Biological and Chemical Sciences, 5, 433-444. https://doi.org/10.4314/ijbcs.v5i2.72073

[16] Agbodjato, N.A., Noumavo, P.A., Baba-Moussa, F., Salami, H.A., Sina, H., Sezan, A., Bankole, H., Adjanohoun, A. and Baba-Moussa, L. (2015) Characterization of Potential Plant Growth Promoting Rhizobacteria Isolated from Maize (Zea mays L.) in Central and Northern Benin (West Africa). Applied and Environmental Soil Science, 2015, Article ID: 901656. https://doi.org/10.1155/2015/901656

[17] MAEP (2016) Catalogue Béninois des Espèces et Variétés végétales (CaBEV), 2016. 4ème trimestre, INRAB/DPVPPAAO/ProCAD/MAEP \& CORAF/WAAPP, Dépôt légal Nº 8982 du 21 octobre 2016, Bibliothèque Nationale (BN) du Bénin, 339.

[18] Connick, W.J., Boyette, C.D. and McAlpine, J.R. (1991) Formulation Mycoherbicides Using a Pasta-Like Process. Biological Control, 1, 282-287. https://doi.org/10.1016/1049-9644(91)90079-F

[19] Walkley, A. and Black, I.A. (1934) An Examination of the Degtjareff Method for Determining Soil Organic Matter and a Proposed Modification of the Chromic Acid 
Titration Method. Soil Science, 37, 29-38.

https://doi.org/10.1097/00010694-193401000-00003

[20] Bray, R.H. and Kurtz, L.T. (1945) Determination of Total Organic and Available Forms of Phosphorus in Soils. Soil Science, 59, 39-45.

https://doi.org/10.1097/00010694-194501000-00006

[21] Thomas, G.W. (1982) Exchangeable Cations. In: Methods of Soil Analysis, Agronomy Monograph 9, ASA and SSSA, Madison, 154-157.

[22] Ruget, F., Bonhomme, R. and Chartier, M. (1996) Estimation simple de la surface foliaire de plantes de maïs en croissance. Agronomie, 16, 553-562. https://doi.org/10.1051/agro:19960903

[23] Yadav, J., Verma, J.P. and Tiwari, K.N. (2010) Effect of Plant Promoting Rhizobacteria on Seed Germination and Plant Growth Chickpea (Cicer arietinum L.) under in Vitro Conditions. Biological Forum, 2, 15-18.

[24] R Core Team (2019) R: A Language and Environment for Statistical Computing. R Foundation for Statistical Computing, Vienna. https://www.R-project.org

[25] Pinheiro, J.C., Bates, D., DebRoy, S.S. and Sarkar, D. (2013) Nlme: Linear and Nonlinear Mixed-Effects Models. R Package Version 31-110.3.1-113.

[26] Alboukadel, K. and Fabian, M. (2017) Factoextra: Extract and Visualize the Results of Multivariate Data Analyses. R Package Version 1.0.5. https://CRAN.R-project.org/package=factoextra

[27] GlèlèKakaï, R., Sodjinou, E. and Fonton, H.N. (2006) Conditions d'Application des Méthodes Statistiques Paramétriques, Bibliothèque Nationale, Bénin.

[28] Husson, F., Josse, J. and Pagès, J. (2010) Principal Component Methods Hierarchical Clustering Partitional Clustering: Why Would We Need to Choose for Visualizing Data? Unpublished Data. http://www.sthda.com/english/upload/hcpc_husson_josse.pdf

[29] Jollife, I.T. (2002) Principal Component Analysis. 2nd Edition, Springer Verlag, New York. https://goo.gl/SB86SR

[30] Abdi, H. and Lynne, J.W. (2010) Principal Component Analysis. WIREs Computational Statistics, 2, 433-459. https://doi.org/10.1002/wics.101 http://staff.ustc.edu.cn/ zwp/teach/MVA/abdi-awPCA2010.pdf

[31] Bidogeza, J.C., Berentsen, B.P.M., De Graaff, J. and Oude-Lansink, A.G.J.M. (2009) A Typology of Farm Households for the Umutara Province in Rwanda. Food Science, 1, 321-335. https://doi.org/10.1007/s12571-009-0029-8

[32] Kumar, A., Maurya, B.R. and Raghuwanshi, R. (2014) Isolation and Characterization of PGPR and Their Effect on Growth, Yield and Nutrient Content in Wheat (Triticum aestivum L.). Biocatalysis and Agricultural Biotechnology, 3, 121-128. https://doi.org/10.1016/j.bcab.2014.08.003

[33] Mejri, D., Gamalero, E. and Souissi, T. (2012) Formulation Development of the Deleterious Rhizobacterium Pseudomonas trivialis X33d for Biocontrol of Brome (Bromus diandrus) in Durum Wheat. Journal of Applied Microbiology, 114, 219-228. https://doi.org/10.1111/jam.12036

[34] Albareda, M., Rodriguez-Navarro, D.N., Camacho, M. and Temprano, F.J. (2008) Alternatives to Peat as a Carrier for Rhizobia Inoculant: Solid and Liquid Formulations. Soil Biology and Biochemistry, 40, 2771-2779. https://doi.org/10.1016/j.soilbio.2008.07.021

[35] Nakkeeran, S., Kavitha, K., Mathiyazhagan, S., Fernando, W.G.D., Chandrasekar, G. and Renukadevi, P. (2004) Induced Systemic Resistance and Plant Growth Promo- 
tion by Pseudomonas chlororaphis Strain PA-23 and Bacillus subtilis Strain CBE4 against Rhizome Rot of Turmeric (Curcuma longa L.). Canadian Journal of Plant Pathology, 26, 417-418.

[36] Hale, L., Luth, M., Kenney, R. and Crowley, D. (2014) Evaluation of Pinewood Biochar as a Carrier of Bacterial Strain Enterobacter cloacae UW5 for Soil Inoculation. Applied Soil Ecology, 84, 192-199. https://doi.org/10.1016/j.apsoil.2014.08.001

[37] Balogoun, I., Saïdou, A., Ahoton, L.E., Adjanohoun, A., Amadji, G.L., Ezui, G., et al. (2013) Détermination des formules d'engrais minéraux et des périodes de semis pour une meilleure production du maïs (Zea mays L.) au Sud et au Centre Bénin. BRAB, 1025-2355 et ISSN en ligne 1840-7099. http://www.slire.net

[38] Mishra, P.K., Bisht, S.C., Bisht, J.K. and Bhatt, J.C. (2012) Cold-Tolerant PGPRs as Bioinoculants for Stress Management. In: Maheshwari, D., Ed., Bacteria in Agrobiology: Stress Management, Springer, Berlin, 95-118.

[39] Kunwar, V.S., Lamichhane, J. and Gauchan, D.P. (2018) Strategies to Improve Phosphorus Availability in a Sustainable Agricultural System. International Journal of Innovative Science and Research Technology, 3, 323-331.

[40] Siddiq, S., Saleem, M.U., Asghar, N., Mahmood, K., Ahmad, K. and Anayat, A. (2018) Comparison of Conventional and Nonconventional Carriers for Bacterial Survival and Plant Growth. Academia Journal of Agricultural Research, 6, 130-134.

[41] Scott, A.H., Garren, P., Ashley, A., Amber, G., Mary, B., Stacy, W., Michael, S. and Richard, F. (2020) Crop Yield, Ferritin and Fe(II) Boosted by Azospirillum brasilense (HM053) in Corn. Agronomy, 10, 394.

http://www.mdpi.com/journal/agronomy https://doi.org/10.3390/agronomy10030394

[42] Al-Erwy, A.S., Al-Toukhy, A. and Bafeel, S.O. (2016) Effect of Chemical, Organic and Bio Fertilizers on Photosynthetic Pigments, Carbohydrates and Minerals of Wheat (Triticum aestivum L.) Irrigated with Sea Water. International Journal of Advanced Research in Biological Sciences, 3, 296-310.

[43] He, W., Adachi, S., Sage, R.F., Ookawa, T. and Hirasawa, T. (2017) Leaf Photosynthetic Rate and Mesophyll Cell Anatomy Changes during Ontogenesis in Backcrossed Indica $\times$ Japonica Rice Inbred Lines. Photosynthesis Research, 134, 27-38. https://doi.org/10.1007/s11120-017-0403-x

[44] Karnwal, A. and Dohroo, A. (2018) Effect of Maize Root Exudates on Indole-3-Acetic Acid Production by Rice Endophytic Bacteria under Influence of L-Tryptophan. F1000Research, 7, 112. https://doi.org/10.12688/f1000research.13644.1

[45] Satyaprakash, M., Nikitha, T., Reddi, E.U.B., Sadhana, B. and Satya Vani, S. (2017) A Review on Phosphorous and Phosphate Solubilising Bacteria and Their Role in Plant Nutrition. International Journal of Current Microbiology and Applied Sciences, 6, 2133-2144. https://doi.org/10.20546/ijcmas.2017.604.251

[46] Khan, A.L., Waqas, M., Kang, S.M., Al-Harrasi, A., Hussain, J., Al-Rawahi, A., Al-Khiziri, S., Ullah, I., Ali, L., Jung, H.Y. and Lee, I.J. (2014) Bacterial Endophyte Sphingomonas sp. LK11 Produces Gibberellins and IAA and Promotes Tomato Plant Growth. Journal of Microbiology, 52, 689-695.

https://doi.org/10.1007/s12275-014-4002-7

[47] Noumavo, A.P., Agbodjato A.N., Gachomo, E.W., Salami, H.A., Baba-Moussa, F., Adjanohoun, A., Kotchoni and Baba-Moussa, L. (2015) Metabolic and Biofungicidal Properties of Maize Rhizobacteria for Growth Promotion and Plant Disease Resistance. African Journal of Biotechnology, 14, 811-819. 
https://doi.org/10.1155/2015/901656

[48] Agbodjato, N.A., Amogou, O., Noumavo, P.A., Dagbenonbakin, G., Hafiz, A.S. Kamirou, R., et al. (2018) Biofertilising, Plant-Stimulating and Biocontrol Potentials of Isolated PGPR Rhizobacteria in Central and Northern Benin. AJMR, 12, 664-672.

[49] Ghaffari, H., Gholizadeh, A., Biabani, A., Fallah, A. and Mohammadian, M. (2018) Effect of Pseudomonas and Azotobacter with Different Levels of Nitrogen on Quantitative Traits and Yield Components of Rice (Variety of Shirudi). Academia Journal of Agricultural Research, 6, 213-221.

[50] Schultz, N., Pereira, W., de Albuquerque Silva, P., Baldani, J.I., Boddey, R.M., Alves, B.J.R., Urquiaga, S. and Reis, V.M. (2017) Yield of Sugarcane Varieties and Their Sugar Quality Grown in Different Soil Types and Inoculated with a Diazotrophic Bacteria Consortium. Plant Production Science, 20, 366-374.

https://doi.org/10.1080/1343943X.2017.1374869

[51] Noel, D.D., Nafan, D., Souleymane, S., Jean-Luc, M.A., Jesus, F. and Baba-Moussa, L. (2016) Combination of Rizhobacteria and Foliar Bio-Fertilizer Accelerating Maize and Soybean Crop Plants Growth Process in Arid Soil. Academia Journal of Agricultural Research, 4, 446-456.

[52] Akhtar, N., Naveed, M., Khalid, M., Ahmad, N., Rizwan, M. and Siddique, S. (2018) Effect of Bacterial Consortia on Growth and Yield of Maize Grown in Fusarium Infested Soil. Soil \& Environment, 37, 35-44. https://doi.org/10.25252/SE/18/872

[53] Paredes-Villanueva, J., Del Rosario, J.L., Urcia Pulido, M.M. and Zavaleta-Armas, J.C. (2020) Plant Growth Promoter Collection of Gluconacetobacter diazotrophicus from Northern Coast of Peru. Scientia Agropecuaria, 11, 15-21. https://doi.org/10.17268/sci.agropecu.2020.01.02

[54] Aquino, J.P.A., Macedo Junior, F.B., Antunes, J.E.L., Figueiredo, M.V.B., Alcantara Neto, F. and Araujo, A.S.F. (2019) Plant Growth-Promoting Endophytic Bacteria on Maize and Sorghum. Pesquisa Agropecuaria Tropical (Agricultural Research in the Tropics), 49, 56241-56241. https://doi.org/10.1590/1983-40632019v4956241

[55] Emami, S., Alikhani, H.A., Pourbabaei, A.A., Etesami, H., Motashare Zadeh, B. and Sarmadian, F. (2018) Improved Growth and Nutrient Acquisition of Wheat Genotypes in Phosphorus Deficient Soils by Plant Growth-Promoting Rhizospheric and Endophytic Bacteria. Soil Science and Plant Nutrition, 64, 719-727. https://doi.org/10.1080/00380768.2018.1510284

[56] Diarrassouba, N., Dago, D.N., Soro, S., Fofana, I.J., Silué, S. and Coulibaly, A. (2015) Multi-Variant Statistical Analysis Evaluating the Impact of Rhizobacteria ( $P$. fluorescens) on Growth and Yield Parameters of Two Varieties of Maize (Zea mays. L). International Journal of Contemporary Applied Sciences, 2, 206-224. 